\title{
Factores que influyen en el uso de servicios de salud por parte de los jóvenes. Caso Universidad Jorge Tadeo Lozano, sede Bogotá
}

\author{
Factors that influence the use of health services by young people. Case of Jorge Tadeo Lozano University, Bogotá \\ headquarters
}

Yorley Quiroga-Otálora1* orcid.org/0000-0003-3810-8802

Leandro González-Támara' orcid.org/0000-0002-9870-2312

1 Universidad Jorge Tadeo Lozano. Bogotá, Colombia

Quiroga-Otálora Y. Factores que influyen en el uso de servicios de salud por parte de los jóvenes. Caso Universidad Jorge Tadeo Lozano, sede Bogotá. Univ. Salud. 2019;21(2):141-151. DOI: http://dx.doi.org/10.22267/rus.192102.148

\section{Resumen}

Introducción: Las universidades tienen constitucionalmente consagrado un principio de autonomía que les permite desarrollar servicios de salud. Objetivo: Determinar los factores por los que los jóvenes utiliza los servicios de salud de la Universidad Jorge Tadeo Lozano. Materiales y métodos: Se analizó estudiantes de pregrado entre 16 y 27 años, que usaron los servicios de salud al menos una ocasión en el periodo comprendido entre el 13 de enero de 2016 al 31 de julio de 2017 a través de una encuesta de 26 preguntas. Resultados: Los costos, hábitos, profesionales y entorno de los jóvenes, además de las campañas de promoción y prevención de salud fueron determinantes para el uso de los servicios. Conclusión: Una política universitaria que promueva el uso de los servicios asistenciales que brindan los centros educativos, fomenta la creación de una cultura del autocuidado y un cambio estructural en la construcción de un estilo de vida saludable en los estudiantes, lo que no implica prescindir del sistema General de Seguridad Social en Salud.

Palabras clave: Adulto joven; adolescente; servicios de salud para estudiantes; promoción de la salud. (Fuente: DeCS, Bireme).

\begin{abstract}
Introduction: Based on the Colombian Constitution, universities have the autonomy to develop their own healthcare service programs. Objective: To determine the factors by which young people use the healthcare services provided by the Jorge Tadeo Lozano University. Materials and methods: Using a 26-question survey, we interviewed students aged between 16 to 27 years, who have used the university healthcare facilities at least once during the period of January 13 to July 312017. Results: The key factors for using healthcare services were cost, habits, health professionals, environment and health promotion and prevention campaigns. Conclusion: A university policy that promotes the use of medical assistance programs provided by educational institutions encourages the establishment of a self-care culture and a structural change in the development of a healthy student lifestyle. A change that does not exclude students from using the General Social Security in Health System.
\end{abstract}

Key words: Young adult; adolescent; student health services; health promotion. (Source: DeCS, Bireme).

\footnotetext{
*Autor de correspondencia

Yorley Quiroga Otálora

e-mail: yorley.quiroga@utadeo.edu.co
} 


\section{Introducción}

La salud en Colombia es un derecho fundamental, para todas las personas que habitan el territorio y está regulado por el Sistema General de Seguridad Social en Salud (SGSSS), los servicios son prestados a toda la población por medio de entidades públicas y privadas, no obstante se identifica tres barreras de acceso al Sistema de Salud: socioculturales, institucionales y políticas, nacionales o territoriales; todas formadas por diferentes categorías 0 variables ${ }^{(1)}$.

En el país uno de los limitantes de acceso a la salud es el gasto de bolsillo, con mayor impacto en la población que no labora, como es el caso de los jóvenes estudiantes universitarios que, por su rol, aún dependen económicamente de sus padres o acudientes, esto se influye en la tasa de desempleo $(17,0 \%)$, de ocupación $(47,7 \%)$ y de inactividad $(42,5 \%)^{(2)}$.

Los estudiantes universitarios al no tener una fuente de ingresos constante presentan dificultad para poder comprar medicamentos y dar continuidad a los tratamientos. En el país los medicamentos son entregados al $54 \%$ de usuarios que pertenecen al régimen contributivo ${ }^{(3,4)}$.

Otra variable que genera un impacto significativo en el uso de los servicios de salud es la información, si esta es deficiente los individuos estarán en una situación de desventaja al desconocer sus deberes y derechos, este punto está definido por factores institucionales que afectan la comunicación. Pero al analizar los aspectos culturales, estos tienen un papel relevante en la creencia y mitos con respecto a los hábitos en salud, que pueden hacer que el individuo acuda a personas no idóneas como los llamados: "yerbateros, teguas o sobanderos", quienes ofrecen tratamientos no convencionales poniendo en riesgo la salud de una comunidad. Otro ejemplo de esto son las concepciones que tienen las personas sobre los hábitos sexuales especialmente en la juventud(5).

Los aspectos culturales algunas veces influyen en los hábitos de los individuos y en esta población se reflejan en enfermedades como depresión, anemia, VIH/sida, lesiones auto provocadas, dolores en la espalda y el cuello, diarrea, trastornos de ansiedad, asma e infecciones de las vías respiratorias inferiores y traumatismos causados por accidentes de tránsito(6).

El gobierno nacional conocedor de la problemática descrita ha diseñado políticas encaminadas a la promoción de la salud en la población juvenil. Para ello se ha desarrollado el programa de servicios amigables, entendidos estos como "aquellos servicios en los cuales adolescentes y jóvenes encuentran oportunidad de salud agradable, cualquiera sea su demanda, para ellos y sus familias, gracias al vínculo que se establece entre usuarios con el proveedor de salud, y por la calidad de sus intervenciones"(7). Por medio de diversos estudios de evaluaciones de impacto, se ha llegado a la conclusión que aquellos adolescentes que interactúan con éstos se vuelven más conocedores de los servicios de salud, aumentando la probabilidad de asistir a un médico general, una leve disminución en los comportamientos de riesgo con la incorporación de algunos hábitos preventivos relativos a la educación sexual y reproductiva(7-9).

Los servicios amigables fueron adoptados en el año 2006 como un modelo orientado a jóvenes y adolescentes con énfasis en prevención y atención de la salud sexual y reproductiva bajo tres modalidades: consulta diferenciada, unidades de salud y centro de salud amigable $(7,10)$; desde un comienzo se ha visto una baja aplicación por los entes públicos, pero se puede identificar una mayor implementación de este modelo en las universidades del país desde el enfoque de la prevención y promoción de la salud(11).

Por otra parte, las universidades han implementado programas de bienestar de carácter obligatorio en el país desde el año 1992, a través del artículo 117 de la Ley 30 , en el en la cual se indica que "las instituciones de Educación Superior deben adelantar programas de bienestar entendidos como el conjunto de actividades orientadas al desarrollo físico, psico-afectivo, espiritual y social de los estudiantes, docentes y personal administrativo". El bienestar incluye diversas variables y términos como lo determina el Consejo Nacional de Educación (CESU) el cual dice que "El bienestar en las instituciones de educación superior es una función de carácter estratégico y transversal que comprende las políticas institucionales, los procesos y las prácticas, así como una cultura institucional que promueve la corresponsabilidad de los actores que conviven en el contexto de la vida institucional para favorecer la 
formación integral, el pleno desarrollo de las capacidades humanas y la construcción de comunidad. El desarrollo humano incluye todos los recursos necesarios para disfrutar de una vida prolongada, saludable y digna"(12).

En la segunda definición se logra observar la incorporación de la salud como un factor necesario para el bienestar, este es un aspecto fundamental que puede cambiar las vidas de los jóvenes, por ejemplo en el área de la sexualidad los embarazos no deseados, que tienen un gran impacto en la vida del joven, dado que genera una interrupción o cambio en los proyectos de vida, siendo alguna de las opciones el abandonar los estudios para empezar a realizar otras actividades de mayor prioridad como el trabajo, y cuidado del niño, entre otros. Sin embargo, al informar, implementar y desarrollar prácticas preventivas o buenas conductas de salud se puede cambiar el bienestar de los jóvenes radicalmente en el corto y largo plazo(13). Es así como los programas de bienestar y los servicios de salud amigables comparten el propósito de la promoción de la salud en la población joven del país vinculada a las instituciones de educación superior, como un mecanismo de responsabilidad social.

Al observar la problemática que viven los jóvenes para acceder a los servicios de salud, se decide analizar los motivos por los cuales utilizan los servicios asistenciales de la Universidad Jorge Tadeo Lozano (UJTL), clasificándolos en tres categorías:

1. Barreras socioculturales: Identifican el entorno del individuo además de los aspectos culturales. Incluyen los costos directos e indirectos en la obtención de los servicios de salud, el transporte entendido como el tiempo de desplazamiento, género, hábitos sexuales, la realización de actividad física, consumo de alcohol, tabaquismo y la alimentación(1).

2. Barreras de las instituciones: Compuestas de la oferta y calidad de los servicios, recursos humanos, procesos administrativos, infraestructura y participación; esta última busca lograr identificar el acceso a la información(1).

3. Barreras políticas: Se ven reflejadas en las actividades desempeñadas por las instituciones, quienes actúan como agentes que velan porque se brinde una adecuada oferta de servicios(1).
Los servicios asistenciales son un componente importante dentro de las acciones de la UJTL para el bienestar de los estudiantes(14). En el contexto institucional, la Universidad cuenta con la Dirección de Bienestar Universitario, adscrita a la Vicerrectoría Académica; a su vez esta Dirección cuenta con tres jefaturas: a) Centro de Arte y Cultura, b) Deportes, y c) Servicios asistenciales. La investigación se centra en el área de servicios asistenciales por estar relacionada con la salud integral de la comunidad institucional y ser la encargada de prestar servicios de salud en medicina, odontología, nutrición, psicología y fisioterapia. Según el Proyecto Educativo Institucional (PEI), su objetivo fundamental es fomentar en la comunidad la promoción de estilos de vida saludable, buscando que las personas asuman de forma responsable su salud, con acciones de autocuidado, logrando una mejor calidad de vida en la UJTL.

Las características generales de la prestación de los servicios de salud en la UJTL son la gratuidad y la oportunidad de acceso a las citas a los diferentes servicios. Existen programas y campañas que se desarrollan como: talleres de técnicas de relajación y de estudio, "atrévete a soñar", campañas de salud oral, programa preventivo de lesiones osteomusculares en el gimnasio, "espalda sana", "que tus abdominales no te depriman", "Tadeo te cuida", "Tadeo sin humo", asesoría en planificación familiar, "charlas sin tapujos" (Plan Estratégico 2015-2020) entre otras que están en proceso de implementación con un direccionamiento novedoso enfocado a la permanencia del estudiante.

La UJTL, acogiendo la normatividad vigente relativa a la prevención y promoción en salud de la población adolescente y juvenil, cuenta con los servicios de bienestar universitario y servicios asistenciales. Por lo tanto, el estudio realizado se centra en los jóvenes estudiantes de pregrado seccional Bogotá y tiene como objetivo determinar los factores por los cuales utilizan los servicios de salud de la Universidad Jorge Tadeo Lozano.

\section{Materiales y métodos}

Investigación es de tipo cuantitativo descriptivo de corte transversal, que consistió en seis fases:

La primera fase consistió en la recolección de información primaria de la dependencia de servicios 
asistenciales de la UJTL, la cual cuenta con un registro de historias clínicas en el programa HOSVITAL de toda la población atendida en alguno de los servicios de salud: medicina, odontología, nutrición, psicología y fisioterapia. Este registro cuenta con datos de los pacientes tales como información personal y académica, servicio consultado, motivo de consulta, antecedentes personales, familiares, evaluación, diagnóstico y tratamiento a seguir. Esta base de datos es una fuente de información estadística que permite realizar una aproximación para la identificación de los factores que influyen en la utilización de los servicios y también establecer un marco de muestreo probabilístico para la realización de encuestas sin consultar la historia clínica de los estudiantes.

Para la segunda fase se procedió a la elaborar una encuesta y una prueba piloto a tres estudiantes incluidos en el marco muestral. Con base en esta prueba se modificaron las preguntas de las características económicas de los individuos. La encuesta contó con 26 preguntas divididas así: a) demografía; b) utilización de servicios asistenciales y c) estilos de vida.

En la tercera fase se realizó una muestra probabilística, estratificada con probabilidades finales desiguales; es probabilística porque cada estudiante-paciente tiene una posibilidad de selección superior a cero. Este tipo de muestra tiene la ventaja de poder establecer a priori en forma aproximada la precisión deseada o esperada en las estimaciones, y calcular a posteriori la precisión efectivamente observada en los resultados. El tamaño de muestra fue calculado asumiendo un error relativo no mayor al $10 \%$ y un efecto de conglomeración de uno. El universo estaba conformado por 2.700 individuos desagregados en 5 especialidades (209 en fisioterapia, 1.709 en medicina, 274 en nutrición, 485 en odontología y 437 en psicología), es decir, un diseño estratificado por especialidad en donde las unidades de muestreo fueron los estudiantes con edades entre los 16 y 27 años. El tamaño de muestra fue de 196 encuestas. Distribuidas de la siguiente manera: fisioterapia 36, medicina 43, nutrición 37 , odontología 40 y psicología 40. Los factores de expansión en las muestras de fisioterapia, medicina, nutrición, odontología y psicología fueron 5,81; 39,$74 ; 7,41 ; 12,13$ y 10,93 respectivamente.

Se decidió centrar el análisis en estudiantes jóvenes de pregrado, de edades entre 16 y 27 años para la elaboración de estadísticas descriptivas, y de 18 a 27 años para la aplicación de la encuesta a usuarios en al menos una ocasión de uno de los servicios de salud entre el 13 de enero de 2016 al el 31 de julio de 2017 y matriculados en el segundo semestre de 2017.

Para la cuarta fase, se seleccionó un universo de estudiantes-pacientes matriculados en la Universidad y que iniciaron actividades académicas en agosto del 2017 todos de edades entre los 18 y 27 años. La selección se realizó utilizando un generador de números aleatorios de una distribución de probabilidad uniforme y luego un coordinado negativo.

En la quinta fase se implementó la encuesta y el consentimiento informado, bajo las siguientes modalidades: forma personal $(32,1 \%)$ y virtual con Google forms $(67,9 \%)$, siendo consolidada la información en un formato de Excel.

En la sexta fase se analizaron los datos con el programa SPSS, realizando estadísticas descriptivas y pruebas de independencia Chi cuadrado para determinar las variables que más influyen en la utilización de los servicios asistenciales.

Se utilizó un muestreo probabilístico para analizar los determinantes de acceso a los servicios de salud (fisioterapia, medicina, nutrición, odontología y psicología) de los estudiantes. Todas las estadísticas que intervinieron en el cálculo del tamaño de muestra: edad, especialidad solicitada, fecha de solicitud del servicio, etc. se basan en la información obtenida de la base de datos de los Servicios Asistenciales de la UJTL que cuenta con 12.301 registros, y que informa sobre la fecha del servicio, el profesional tratante, la identificación del paciente (sexo, programa), el detalle de la consulta y su diagnóstico.

\section{Consideraciones éticas}

Según la Resolución 8430 de 1993 del Ministerio de Salud por la cual se establecen las normas científicas, técnicas y administrativas para la investigación en salud este estudio se clasifica en la categoría "sin riesgo", teniendo en cuenta que es un estudio sin intervención y que los datos se obtuvieron de la revisión de la información del departamento de mercadeo, planeación y del sistema de historias clínicas de la Jorge Tadeo Lozano de servicios asistenciales. La investigación contó con el aval ético 
de la institución educativa, todos los entrevistados participaron de manera voluntaria y se les dio la oportunidad de rechazar o aceptar su inclusión en el estudio. Todos los participantes diligenciaron $\mathrm{y}$ firmaron un formato de consentimiento informado; ningún menor de edad participó en el desarrollo de la encuesta.

\section{Resultados}

De acuerdo a la información de los Servicios Asistenciales durante el periodo considerado se realizaron 12.301 consultas principalmente de medicina $(40,8 \%)$, psicología $(15,5 \%)$, odontología $(15,2 \%)$, nutrición $(14,4 \%)$ y fisioterapia $(14,2 \%)$. Estas 12.301 consultas correspondieron a 4.946 personas, es decir, estos estudiantes solicitaron uno o más servicios de una o más especialidades; de ellos, se contactó tan solo a 3.114, porque los demás no estaban matriculadas para el periodo académico de agosto a noviembre de 2017. Para la aplicación del formulario estructurado se incorporó a la base de datos un filtro por edad resultando 2.700 estudiantes entre 18 y 27 años. En la encuesta aplicada a los 196 estudiantes de pregrado, se encontró los datos que se presentan a continuación según las tres partes de la encuesta:

\section{a) Demografía}

En la Figura 1 se muestra distribución de la edad de esta población respecto a la utilización de los servicios asistenciales.

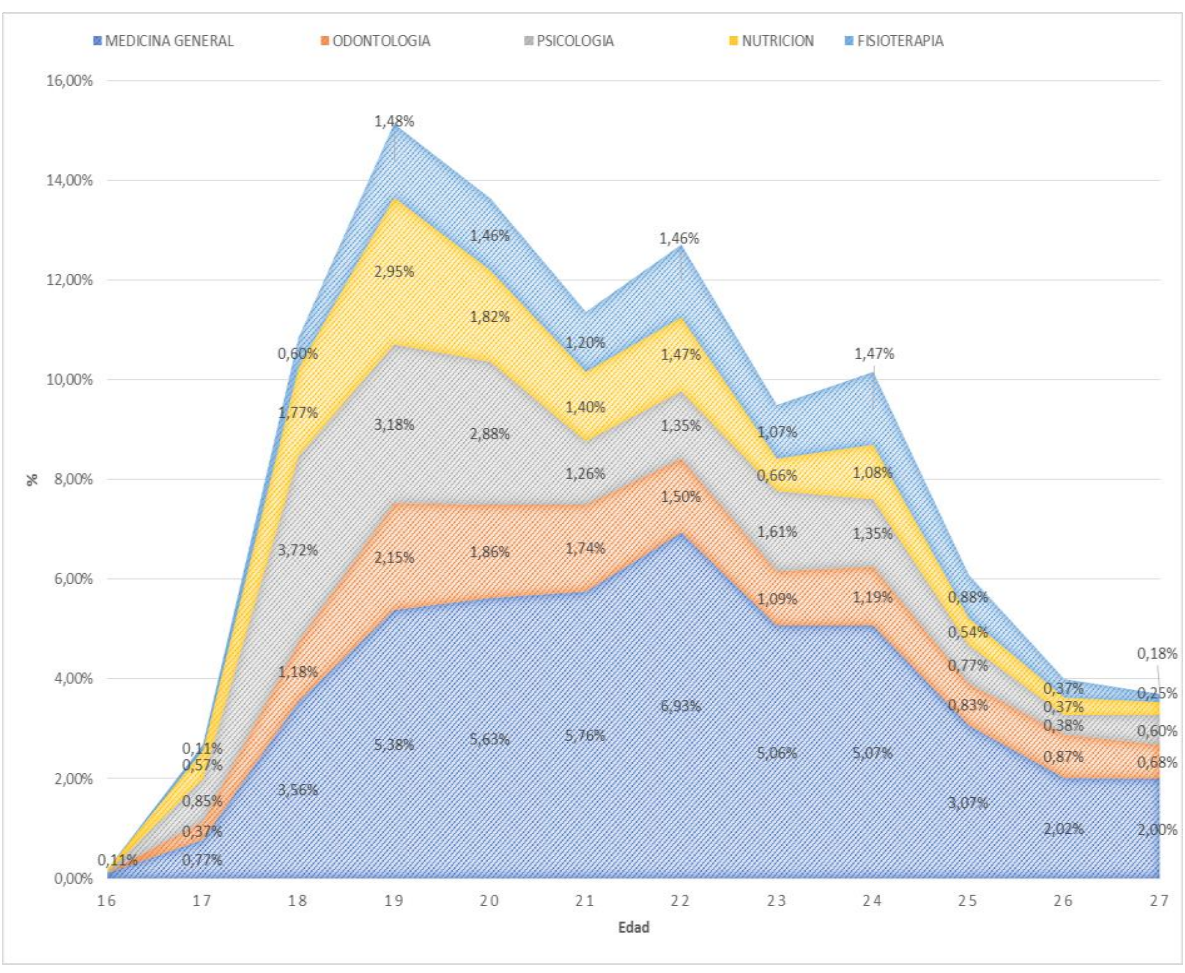

Figura 1. Distribución de la edad por uso de servicios

El 59,6\% de los individuos de la muestra son mujeres y el $97,2 \%$ afirma estar soltero.

Referente a la demanda de los servicios prestados, el programa de Ingeniería Química generó la mayor utilización $(15,2 \%$,$) uno de los factores que puede$ explicar este comportamiento es debido a que este programa cuenta con el mayor número de estudiantes para el periodo de estudio. El $99,6 \%$ se encuentra afiliado al Sistema de Salud bajo cualquier modalidad.
En la Tabla 1 se muestran algunas de las características de las personas participantes.

Respecto a la situación laboral se evidenció que el $57,8 \%$ de estudiantes no trabaja, el $12,8 \%$ trabaja tiempo completo, el 10,8\% únicamente en vacaciones, $8,0 \%$ en fin de semana, $7,9 \%$ medio tiempo y $2,7 \%$ tiempo parcial. El $22,5 \%$ de los individuos que trabajan, manifiestan que si dejaran de desempeñar su actividad económica, se verían obligados a suspender sus estudios. 
Tabla 1. Variables descriptivas de la población

\begin{tabular}{lr}
\hline \multicolumn{1}{c}{ Variable } & \% \\
\hline Personas con quien convive & \\
Con ambos padres & 41,5 \\
Sólo con el padre & 5,4 \\
Sólo con la madre & 22,5 \\
Otros familiares & 12,4 \\
Otros no familiares & 8,0 \\
Con la pareja & 2,8 \\
Solo & 7,4 \\
Estado de la vivienda & \\
Arriendo & 54,9 \\
Propia & 31,7 \\
Se encuentra pagándola & 12,1 \\
Usufructo & 1,3 \\
Situación económica & \\
Aporta dinero al hogar & 17,7 \\
Cambio de residencia por ingresar a la & 19,5 \\
universidad & \\
Tiempo de desplazamiento & \\
Menos de 30 minutos & 20,5 \\
30 - 60 minutos & 42,5 \\
Más de 60 minutos & 34,3 \\
\hline
\end{tabular}

\section{b) Servicios asistenciales}

La información sobre servicios de salud más utilizados en la UJTL y sobre los diagnósticos más frecuentes se resume en la Tabla 2 .

Entre los motivos del por qué usan los servicios de salud universitarios se encontró que una de las mayores motivaciones es que no generan un costo al estudiante $(24,8 \%)$, la capacidad de poder acceder a varios servicios sin la necesidad de autorizaciones $(16,5 \%)$, el poder utilizar los servicios en su tiempo libre $(12,6 \%)$, por una oferta oportuna y profesionales idóneos $(8,4 \%)$, disponibilidad de la agenda $(5,9 \%)$, porque los servicios cuentan con tecnología de punta $(7,0 \%)$, porque la incapacidad médica es un soporte válido para la inasistencia a clase no mayor a dos días $(4,8 \%)$.

Respecto a la inasistencia a la consulta se encontró: odontología $(11,7 \%)$, nutrición $(5,3 \%)$, medicina general $(1,6 \%)$, psicología $(4,0 \%)$, en fisioterapia $(2,2 \%)$; siendo los principales motivos para su incumplimiento el no tener tiempo durante su jornada académica $(30,2 \%)$, el tiempo de espera para la atención $(18,7 \%)$, la cantidad de trámites a realizar $(12,7 \%)$, el considerar su estado de salud como bueno $(8,1 \%)$, la mala atención por parte del personal administrativo $(2,7 \%)$, la presentación de diversos problemas $(26,1 \%)$.

Tabla 2. Servicios y diagnósticos de salud en la UJTL

\begin{tabular}{|c|c|}
\hline Servicios de salud más utilizados en la UJTL & $\%$ \\
\hline Medicina general & 56,0 \\
\hline Psicología & 14,5 \\
\hline Odontología & 13,8 \\
\hline Nutrición & 8,9 \\
\hline Fisioterapia & 6,8 \\
\hline \multicolumn{2}{|l|}{ Diagnósticos más frecuentes en la UJTL } \\
\hline $\begin{array}{l}\text { Problemas relacionados con la dieta y hábitos } \\
\text { alimentarios inapropiados }\end{array}$ & 8,9 \\
\hline Rinofaringitis viral aguda "resfriado común" & 6,9 \\
\hline Gingivitis crónica & 6,7 \\
\hline $\begin{array}{l}\text { Problemas relacionados con el ajuste a las } \\
\text { transiciones del ciclo vital }\end{array}$ & 6,1 \\
\hline Esguinces y torceduras del tobillo & 3,4 \\
\hline $\begin{array}{l}\text { Problemas relacionados con habilidades sociales } \\
\text { inadecuadas no clasificadas en otra parte }\end{array}$ & 2,4 \\
\hline Caries dental & 2,2 \\
\hline $\begin{array}{l}\text { Diarrea y gastroenteritis de presunto origen } \\
\text { infeccioso }\end{array}$ & 1,8 \\
\hline Lumbago no específico & 1,7 \\
\hline Hipoglicemias & 1,5 \\
\hline $\begin{array}{l}\text { Examen para participación en competencias } \\
\text { deportivas }\end{array}$ & 1,3 \\
\hline Hipo mineralización localizada & 1,3 \\
\hline $\begin{array}{l}\text { Trastorno de la personalidad emocionalmente } \\
\text { inestable }\end{array}$ & 1,2 \\
\hline Problemas relacionados con la falta de ejercicio & 1,2 \\
\hline $\begin{array}{l}\text { Problemas relacionados con el bajo rendimiento } \\
\text { académico }\end{array}$ & 1,2 \\
\hline Síndrome del colon irritable sin diarrea & 1,2 \\
\hline Desgarro o distensión muscular & 1,1 \\
\hline Dispepsia & 1,1 \\
\hline Otras caries dentales & 1,1 \\
\hline Mareo y desvanecimiento & 1,0 \\
\hline Cefalea & 0,9 \\
\hline Otras & 45,2 \\
\hline
\end{tabular}

\section{c) Estilos de vida}

Los jóvenes realizan actividad física en la semana: una vez $(19,1 \%)$, dos veces $(16,1 \%)$, tres veces $(12,8 \%)$, cuatro veces $(16,0 \%)$, cinco veces $(5,5 \%)$, seis veces $(2,7 \%)$, más de seis veces $(0,7 \%)$ y siendo la más frecuente el no realizarla $(27,2 \%)$. Respecto al consumo de alcohol indicaron que consumen en el mes: una vez $(46,0 \%)$, de dos a tres $(7,3 \%)$, de cuatro a cinco $(1,5 \%)$ y nunca han consumido $(45,1 \%)$. 
En cuanto al consumo de tabaco durante la semana la respuesta predominante fue el nunca haber fumado $(72,5 \%)$, seguido de una vez $(7,9 \%)$, dos a tres $(6,0 \%)$, más de $7(6,0 \%)$. Con relación al número de comidas durante su jornada estudiantil el $(38,2 \%)$ realiza tres comidas, $(29,8 \%)$ dos comidas, y una comida el $(23,4 \%), y$ más de tres comidas $(8,6 \%)$.

En cuanto al uso de preservativos durante las relaciones sexuales, el $(28,3 \%)$ indicó no haber tenido relaciones sexuales, el de las personas que tienen una vida sexual activa, usan siempre el preservativo $(51,3 \%)$, casi siempre $(11,2 \%)$, algunas veces $(6,4 \%)$ y nunca $(2,8 \%)$.

Por último, de los estudiantes analizados: el 2,1\% consume sustancias psicoactivas y en cuanto a la pregunta sobre el conocimiento de campañas de promoción y prevención el 35,0\% afirma conocerlas.

Para cumplir con el objetivo de la sexta fase del estudio, se procedió a realizar un análisis estadístico para determinar cuáles son las principales variables que influyen en la decisión de usar los servicios por parte de los estudiantes.

\section{Variables influyentes en la utilización de los servicios asistenciales}

A continuación se muestra la dependencia entre diferentes tipos de variables (estado civil, personas con quien convive el estudiante, tipo de vivienda, tiempo de desplazamiento, actividad física, razones económica, tipo de trabajo, condición de fumador, consumo de alcohol, número de comidas en horas de estudio en la universidad, conocimiento de campañas de promoción), con relación a si utilizan o no los diferentes tipos de servicios de salud ofrecidos por la universidad, para poder analizar los factores influyentes en el uso de estos.

Se realizaron pruebas Chi cuadrado con un nivel de confianza superior al $95 \%$ para analizar la dependencia entre cada una de las variables y aquella que recoge la información acerca de la utilización de cada uno de los servicios asistenciales(16). Por ejemplo la variable trabajo fue analizada en dos grupos así: quienes no trabajan o solo lo hacen en periodos de vacaciones y quienes trabajan durante el periodo académico (tiempo completo, parcial o fines de semana), y se examinó su dependencia con la utilización del servicio de medicina. La prueba Chi cuadrado realizada tuvo como hipótesis nula la independencia entre dos variables, es decir, que las proporciones de personas que utilizan este servicio son similares entre quienes no trabajan durante el periodo académico y quienes si lo hacen.

De acuerdo a la prueba $\mathrm{Chi}^{2}$ se observó que algunas variables influyen en la utilización de los servicios, estas son resaltadas en casillas de color naranja en la Tabla 3.

Se observa que el estado civil, las personas con quienes convive el estudiante y el tipo de vivienda son variables influyentes en la utilización de todos los servicios. En particular la condición de soltero en una variable que ha sido reportada en la literatura como muy influyente en el desarrollo de la vida universitaria en Bogotá(14). Con respecto a la misma prueba se evidencia un grado de importancia menor de las variables tiempo de desplazamiento, número de comidas realizadas en la Universidad, el conocimiento de las campañas de promoción y prevención de salud y la edad porque resultaron ser dependientes con un número menor de servicios. Por último, se observa que variables como el consumo de alcohol o la realización de actividad física son menos influyentes en la utilización de los servicios asistenciales en la Universidad.

La última fila de la Tabla 2 contiene los resultados de la influencia de la variable edad, que fue estudiada utilizando un análisis de varianza comparando las edades promedio de quienes utilizan y no utilizan los diferentes servicios. Se encontró que quienes tienen una edad significativamente mayor, utilizan los servicios de medicina y fisioterapia; quienes tienen una edad significativamente menor utilizan los servicios de odontología y psicología; y por último, se observa que no existe diferencia significativa en la edad promedio de quienes utilizan o no, el servicio de nutrición. Según la Tabla 2, aquellos casos en donde se probó la dependencia entre cada par de variables, los niveles de confianza de estas pruebas de hipótesis son siempre superiores al 95\% (valores p menores a $0,05)$. 
Tabla 3. Variables influyentes en la utilización de los servicios asistenciales

\begin{tabular}{|c|c|c|c|c|c|}
\hline Variable & Medicina & Odontología & Nutrición & Psicología & Fisioterapia \\
\hline Estado civil & $<0,0001$ & $<0,0001$ & $<0,0001$ & $<0,0001$ & 0,007 \\
\hline Personas con quien convive & $<0,0001$ & $<0,0001$ & $<0,0001$ & $<0,0001$ & 0,007 \\
\hline Tiempo de desplazamiento & 0,253 & $<0,0001$ & $<0,0001$ & $<0,0001$ & 0,007 \\
\hline Actividad física & 0,970 & 0,047 & 0,029 & $<0,0001$ & 0,174 \\
\hline Razones económica & 0,026 & $<0,0001$ & $<0,0001$ & $<0,0001$ & 0,243 \\
\hline Condición de fumador & $<0,0001$ & $<0,0001$ & 0,003 & 0,932 & 0,311 \\
\hline Consumo de alcohol & 0,040 & 0,180 & 0,062 & 0,470 & $<0,0001$ \\
\hline Número de comidas en la Universidad & 0,983 & $<0,0001$ & $<0,0001$ & $<0,0001$ & $<0,0001$ \\
\hline $\begin{array}{l}\text { Conocimiento de campañas de promoción y } \\
\text { prevención en salud }\end{array}$ & 0,745 & $<0,0001$ & $<0,0001$ & $<0,0001$ & 0,011 \\
\hline
\end{tabular}

Valor p de la prueba Ji cuadrado de independencia entre cada par de variables. (El color naranja indica dependencia significativa)

\section{Discusión}

En Colombia la población tiene la obligación y el derecho a estar afiliado al Sistema General de Seguridad Social en Salud (SGSSS) en cualquiera de sus regímenes, según su capacidad económica. Por esta razón, los estudiantes de la UJTL deben estar afiliados ya sea como cotizantes o beneficiarios al régimen contributivo o subsidiado. Sin embargo, existe un pequeño grupo de estudiantes que no se encuentran afiliados al SGSSS.

Por medio del análisis de los datos, se puede observar que la gratuidad en los servicios asistenciales de la UJTL puede ser una razón para su uso, mientras que en su Entidad Prestadora de Servicios de Salud (EPS), deben cancelar una cuota moderadora o copago, lo cual aumenta el gasto de dinero y como se ha indicado es una de las barreras de acceso identificadas en diversos estudios. Es importante resaltar que otro factor de gran importancia que tiene un impacto significativo en el uso de los servicios es la autonomía que adquieren los jóvenes al ingresar a la universidad, esto se puede observar en la variable de los individuos que viven solos(17).

Se ha demostrado que los padres, especialmente las madres, tienen una fuerte influencia en la utilización de los servicios de salud, puesto que se enmarca una constante preocupación por el bienestar de sus hijos, siendo alguna de las herramientas la implementación de hábitos saludables, asistencia a citas de control médico entre otros, siempre jugando un papel activo y de liderazgo; cuando los hijos dejan el núcleo familiar estos adquieren dichas responsabilidades, como: la de escoger el lugar, profesional y especialidad de salud(18,19). Mientras sí viven con sus padres, estos pueden generar el incentivo a la utilización y dejar al individuo escoger el lugar donde accederá.

Al observar la situación laboral la mayoría de estudiantes no labora, es decir que sus ingresos provienen principalmente de sus padres o terceros que patrocinan sus estudios, por ello sus gastos son limitados y adicionalmente, como se ha demostrado los jóvenes no tienen un fuerte interés en su estado de salud(20-23). En consecuencia deben distribuir una porción de sus ingresos para el uso de los servicios de salud en su EPS, generando una fuerte desmotivación para su uso, puesto que preferirán gastar ese dinero en otro bien o servicio que les produzca mayor felicidad(20,21). Se puede suponer que los individuos que trabajan tiempo parcial tienen más opciones de ajustar sus actividades académicas y laborales, esto les permite fácil acceso a los servicios asistenciales de la UJTL. Aquellos que trabajan tiempo completo tienen mayores limitaciones para el acceso a los servicios asistenciales que no se ofertan en horario nocturno: nutrición, odontología, psicología y fisioterapia. Ya se ha discutido que los estudiantes que desempeñan alguna actividad laboral con una asignación equivalente a un salario mínimo vigente o inferior, presentan una tendencia a sufrir patologías físicas y emocionales(22,23). 
Respecto al tiempo de desplazamiento, el costo que genera el transporte se puede ver relacionado con lo expuesto previamente, dado que movilizarse fuera de las instalaciones a una cita médica ocasiona un costo que no está presupuestado en su diario vivir. Además, el tiempo empleado en la movilización del individuo se convierte en una razón para no usar el servicio, puesto que los jóvenes se caracterizan por querer las cosas con la mayor rapidez posible(24); la posibilidad de dar uso a los servicios mientras están desarrollando actividades académicas produce un impacto positivo hacia el uso porque el individuo ve la facilidad de agendar la cita médica en los espacios libres entre clases(25).

Los estilos de vida tienen un efecto dependiendo del servicio utilizado por parte de los estudiantes. La actividad física influye en la utilización de los servicios de odontología, nutrición y psicología; pero no es significativo en la utilización de los servicios de medicina y fisioterapia. Esto contrasta con lo encontrado en la literatura ya que las consultas de fisioterapia pueden aumentar cuando los estudiantes tienen actividad física regular(26).

En el caso del servicio de nutrición se identificaron problemas de malos hábitos nutricionales, los cuales son generados por consumir pocas comidas saludables y balanceadas durante su jornada estudiantil, debido a la falta de tiempo en sus clases y pocos ingresos monetarios, originando desórdenes alimenticios; este servicio se utiliza principalmente porque el individuo reconoce tener un mal estado de salud y manifiestan un deseo de cambiar sus hábitos para obtener resultados que se reflejen en su cuerpo $^{(9)}$.

Por otra parte, los estudiantes presentan algunas manifestaciones clínicas derivadas del estrés producido por las actividades académicas, las cuales conllevan a que los jóvenes puedan tener dificultad para adaptarse a la universidad(27). El hábito de fumar tiene una relación con problemas de vías respiratorias y cambios dentales, que producen una mayor utilización del servicio de odontología. Con relación al consumo de alcohol, se evidencia que conlleva a un aumento de sufrir lesiones osteomusculares o heridas, pero en el caso de medicina se observa lo contrario lo que hace necesario indagar más sobre este aspecto(28).
La percepción de los jóvenes respecto a algunos servicios, indica que los factores institucionales, como la infraestructura, profesionales y parte administrativa, son importantes para generar una continuidad en los tratamientos. Se encontró que los individuos tienen poco conocimiento de las campañas de promoción y prevención, pero esta variable indica que un mayor conocimiento en esta temática produce un impacto en el aumento de uso; excepto en el caso de medicina, en el cual se puede observar un interés por la incapacidad médica, la cual genera excusas por inasistencia a clase no mayor a dos días por enfermedad. En el caso cuando el evento requiere de mayor tiempo de incapacidad médica, el estudiante es remitido a su Entidad Prestadora de Servicios de Salud.

\section{Conclusiones}

La gratuidad de los servicios de salud al interior de la UJTL, es registrada por los estudiantes como el principal factor para hacer uso de estos; con relación a la segunda variable se resalta la atención amigable y comprensiva de los profesionales, un ambiente visualmente agradable que genera un mayor impacto en el uso. Ambas variables pertenecientes a la categoría institucional que puede ser considerada como la que genera el mayor impacto en el uso, si la planeación y desarrollo de las campañas de promoción y prevención de salud se caracterizan por una comunicación efectiva y apropiada para el público al que va dirigida, esto impactará en estilos de vida saludable. Estas campañas siempre deben ir acompañadas de una evaluación que permita observar si se está dando respuestas a las necesidades de la población.

Los principales diagnósticos de servicios asistenciales de la UJTL se encuentran dolores en la espalda y el cuello, enfermedades diarreicas y los problemas relacionados con el ajuste a las transiciones del ciclo vital (establecer relaciones conmigo mismo y con los demás).

El Área de Servicios Asistenciales de la Universidad Jorge Tadeo Lozano, desempeña un papel importante como mecanismo complementario del Sistema de Salud colombiano, teniendo en cuenta que este se acoge a los Servicios Amigables; en este sentido la oportunidad de acceder a los servicios básicos de salud como: medicina, odontología, nutrición, psicología y fisioterapia, permite a los estudiantes 
completar su formación integral con el objetivo de promover una cultura de salud preventiva que se refleje en una mejor calidad de vida de quienes conforman la comunidad tadeísta.

Es importante gestionar una política universitaria que promueva en los jóvenes el uso de los servicios asistenciales que brindan sus centros educativos, plantear la creación de una cultura del autocuidado y un cambio estructural en la construcción de un estilo de vida saludable en los estudiantes, que no implica prescindir del sistema General de Seguridad Social en Salud. Se logró establecer las variables que tenían una relación con el uso de los servicios asistenciales, pero se sugiere, para estudios posteriores, indagar acerca de cuáles de estas variables tienen mayor impacto en la decisión de los estudiantes de usar los servicios de salud de la universidad.

Desde el punto metodológico se encontró algunas limitaciones técnicas para la aplicación de la encuesta digital, por esta razón, se diligenció de manera personal empleando mayor tiempo en esta fase de la investigación.

\section{Agradecimientos}

A los profesores: Juan Carlos Córdoba, Giovanni Jiménez y al estudiante de maestría Johan Barajas.

\section{Conflicto de intereses}

Los autores declaran que no existen conflictos de interés.

\section{Referencias}

1. Ministerio de la Protección Social, UNFPA. Servicios de Salud Amigables para Adolescentes y Jovenes. $2^{\circ}$ edición. Bogotá: MinProtección Social-IUNFPA; 2008.

2. Departamento Administrativo Nacional de Estadística. Informes especiales de mercado laboral [Internet]. Bogotá: DANE; 2018 [cited 2018 Jul 27]. Available from: https://www.dane.gov.co/files/investigaciones/boletines/ech/ ech/comunicados_de_prensa/Cp_GEIH_feb18_abr18.pdf

3. Morales-Sánchez LG, García-Ubaque JC. Gestión de la crisis del sistema de salud en Bogotá. Rev Salud Pública [Internet]. 2017 [cited 2018 Jul 27];19(1):129-33. Available from: https://doi.org/10.15446/rsap.v19n1.64102

4. Defensoría del Pueblo Colombia. La tutela y los derechos a la salud y a la seguridad social 2014. Bogotá: Imprenta Nacional de Colombia; 2014.

5. Aguirre-Quezada JP. Salud en el Valle de México, embarazo adolescente y la figura política percibida con más autoridad en las entidades federativas. Visor Ciudad [Internet]. 2013;(11):22. Available from: http://www.bibliodigitalibd.senado.gob.mx/bitstream/handle/ 123456789/2086/VC11.pdf?sequence=1\&isAllowed=y
6. Organización Mundial de la Salud. La OMS pide que se preste mayor atención a la salud de los adolescentes [Internet]. Ginebra: OMS; 2014 [cited 2018 Feb 25]. Available from: http://www.who.int/mediacentre/news/releases/2014/focusadolescent-health/es/

7. Quintero-Rondón AP, Rojas-Betancur HM. El embarazo a temprana edad, un análisis desde la perspectiva de madres adolescentes. Rev Virtual Univ Católica del Norte [Internet]. 2015;44:222-37. Available from: http://revistavirtual.ucn.edu.co/index.php/RevistaUCN/article /viewFile/626/1161

8. Vasquez-Dulce HE, Vasquez-Dulce HE. Consumo de cigarrillo tradicional en adolescentes: un grave problema de Salud Pública. Medidas efectivas para su prevención. Rev Med Chil [Internet]. 2018 [cited 2018 Jul 27]; 146(1):122-3. Available from: http://www.scielo.cl/scielo.php?script=sci_arttext\&pid=S0034$98872018000100122 \& \operatorname{lng}=$ en\&nrm=iso\&tlng=en

9. Sánchez-Ojeda MA, Luna-Bertos E De. Hábitos de vida saludable en la población universitaria. Nutr Hosp [Internet]. 2015 [cited 2018 Jul 27];31(5):1910-9. Available from: http://scielo.isciii.es/scielo.php?script=sci_arttext\&pid=S021216112015000500003

10. Andradas-Aragonés E; et al. Actividad física para la salud y reducción del sedentarismo: Recomendaciones para la población [Internet]. Madrid, España: Ministerio de Sanidad, Servicios Sociales e Igualdad; 2015. 28 p. Available from: https://www.msssi.gob.es/profesionales/saludPublica/prevPr omocion/Estrategia/docs/Recomendaciones_ActivFisica_para_l a_Salud.pdf

11. Ara N, Saldaña Y, Fernández N DH. Salud, calidad de vida y entorno universitario en estudiantes mexicanos de una universidad pública. Rev Hacia la Promoción la Salud [Internet]. 2015 [cited 2018 Jul 27];20(2):102-17. Available from: http://www.redalyc.org/html/3091/309143500008/

12. Ministerio de Educación Nacional. Lineamiento de política de bienestar para instituciones de educación superior [Internet]. Bogotá: MinEducación; 2016 [cited 2018 Jul 27]. Available from: https://www.mineducacion.gov.co/1759/articles360314_recurso.pdf

13. World Health Organization. Adolescent Friendly Health Services : An Agenda for Change [Internet]. Geneva, Switzerland: WHO; 2002. 44 p. Available from: https://www.who.int/maternal_child_adolescent/documents/f ch_cah_02_14/en/

14. Barragán-Moreno SP, González-Támara L. La Universidad de Bogotá Jorge Tadeo Lozano en el camino de la retención estudiantil. Primera. Castiblanco JM, editor. Bogotá D.C: Fundación Universidad de Bogotá Jorge Tadeo Lozano; 2015. $181 \mathrm{p}$.

15. Ospina-Botero D. Introducción al muestreo. Bogotá: Universidad Nacional de Colombia; 2001. 262 p.

16. González-Támara L. Análisis exploratorio de datos. Una introducción a la estadística descriptiva y probabilidad. Bogotá: Universidad Jorge Tadeo Lozano; 2017. 340 p.

17. León OD. Políticas sociales, jóvenes y Estado: o el síndrome del padre ausente. Última Década [Internet]. 1999;(11):1-10. Available from: http://www.redalyc.org/articulo.oa?id=19501112

18. Lavielle P, Pineda V, Jáuregui O, Castillo M. Actividad física y sedentarismo: Determinantes sociodemográficos, familiares y su impacto en la salud del adolescente. Rev Salud Pública [Internet]. 2014;16(2):161-72. Available from: http://www.revistas.unal.edu.co/index.php/revsaludpublica/a rticle/view/33329/47680

19. Bernal O, Barbosa S. La nueva reforma a la salud en Colombia: el derecho, el aseguramiento y el sistema de salud. Salud Pública Mex [Internet]. 2015 [cited 2018 Jul 27];57(5):433-40. Available 
from: http://www.medigraphic.com/pdfs/salpubmex/sal2015/sal155j.pdf

20. Rodríguez-Jiménez RM, Velasco-Quintana PJ, Terrón-López J. Construyendo universidades saludables: conciencia corporal y bienestar personal. 2014 [cited 2018 Jul 24];66:207-24. Available from: http://82.223.175.156/bitstream/handle/11268/3753/rie66a 13.pdf? sequence $=2$ \&isAllowed $=y$

21. Moyano-Díaz E. Afrontamiento, salud mental y felicidad bajo huelga y ocupación universitariai. Arq Bras Psicol [Internet]. 2017 [cited 2018 Jul 27];69(2):153-67. Available from: http://www.redalyc.org/articulo.oa?id=229053873011

22. Moreta-Herrera R, Gaibor I, Barrera L. El bienestar psicológico y la satisfacción con la vida como predictores del bienestar social en una muestra de universitarios ecuatorianos. Salud Soc [Internet]. 2017 Oct 6[cited 2018 Jul 27];8(2):172-84. Available from:

http://146.83.115.167/index.php/saludysociedad/article/view $/ 1678 /$ pdf

23. Rubio-Henao RF, Varela-Arevalo MT. Barreras percibidas en jóvenes universitarios para realizar actividad física. Rev Cuba Salud Pública [Internet]. 2016 Mar 28 [cited 2018 Jul 27];42(1):61-9. Available

from:
24. López de Ayala MC, García BC, Alfageme P. Hábitos de búsqueda y confianza en la información online sobre salud entre jóvenes universitarios [Internet]. Madrid: VI Congreso Internacional Latina de Comunicación Social; 2014 [cited 2018 Jul 27]. Available from: http://www.revistalatinacs.org/14SLCS/2014_actas.html

25. Paredes-Samame S. Percepción de los adolescentes de la calidad de atención de los servicios de salud en el Centro Materno Infantil Daniel Alcides Carrión - Villa María del Triunfo, 2017. Repos Inst - UAP [Internet]. 2017 [cited 2018 Jul 27]; Available from: http://repositorio.uap.edu.pe/handle/uap/6476

26. Andradas E, Begoña A, Merino M, Campos P, Ana E, Luciano G, et al. Actividad física para la salud y reducción del sedentarismo [Internet]. Madrid; 2015 [cited 2018 Jul 27]. Available from: http://publicacionesoficiales.boe.es/

27. Álvarez J, Aguilar JM, Lorenzo JJ. La Ansiedad ante los Exámenes en Estudiantes Universitarios: Relaciones con variables personales y académicas. Electron J Res Educ Psychol [Internet]. 2017 Nov 23 [cited 2018 Jul 27];10(26). Available from: http://ojs.ual.es/ojs/index.php/EJREP/article/view/1497

28. Navarrete-Cruz JL. Consumo de Alcohol y Conductas Agresivas en Adolescentes Estudiantes de la Unidad Educativa "Atahualpa" del Cantón Ambato. Ambato: Universidad Técnica de Ambato; 2016 [cited 2018 Jul 27]; Available from: http://repositorio.uta.edu.ec/handle/123456789/23448 